\title{
Analysis of Small Field Dosimetry for 15 MV Photon Beams
}

\author{
Yonca YAHŞI \\ Şişli Hamidiye Etfal Education and Research Hospital, Department of Radiation Oncology, \\ Istanbul, Turkey \\ yoncayahsi@mynet.com \\ Meral KURT \\ Uludağ University School Of Medicine, Department of Radiation Oncology, Bursa, Turkey

\section{Lütfi ÖZKAN} \\ Denizli Private Health Hospital, Radiation Oncology Department, Denizli, Turkey
}

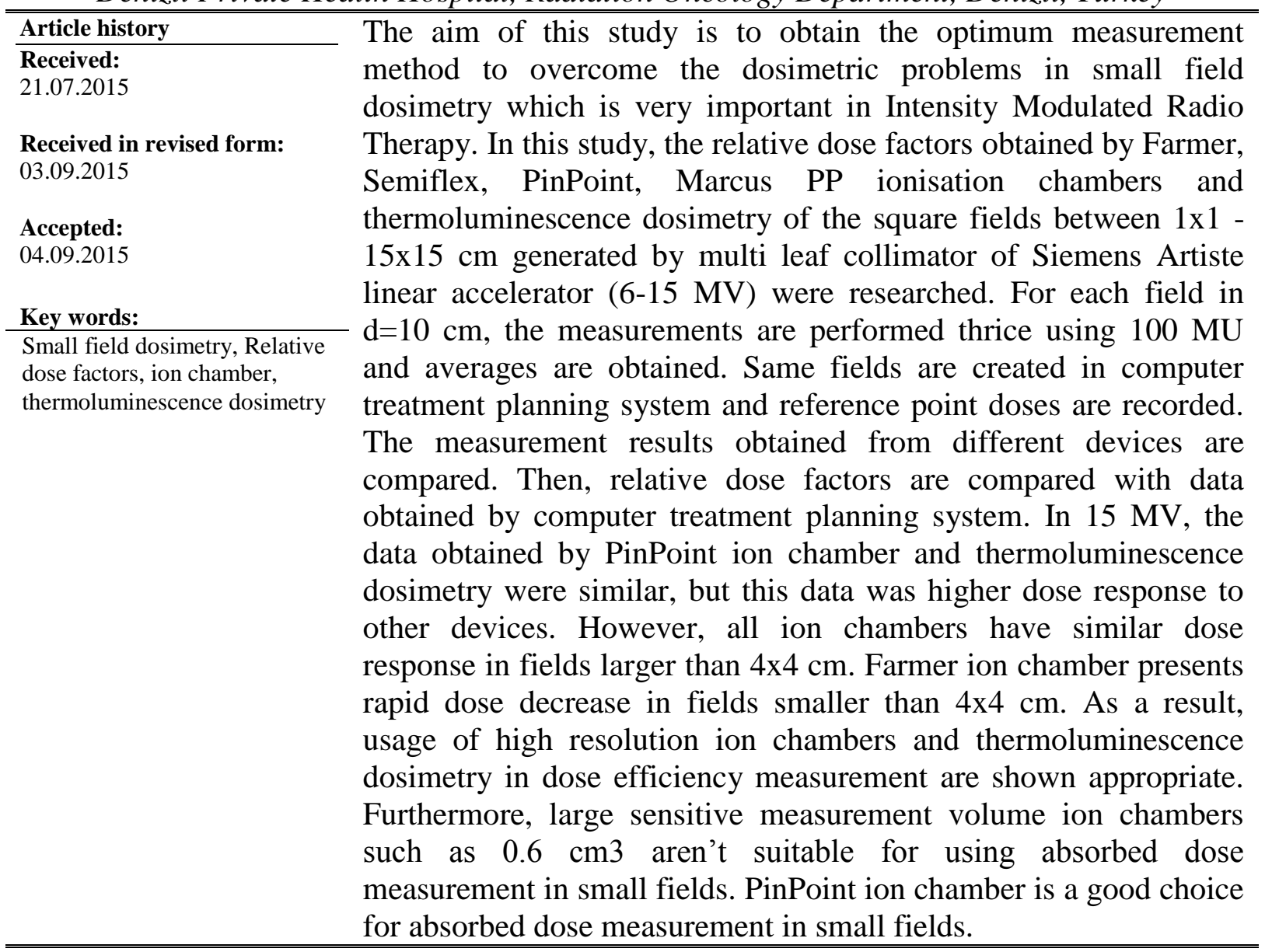

*Correspondence:yoncayahsi@mynet.com 


\section{Introduction}

The fundamental target in radiotherapy is to give the suitable homogeneous dose to the target volume by keeping the normal tissue dose minimum. It could not be possible with common conventional techniques nowadays. The most suitable (conformal) dose distribution in target could be obtained by Intensity Modulated Radio Therapy (IMRT) that has developed in recent years. Since this theory technique provides the opportunity to protect the healthy tissues in a better way, it allows to reach higher doses in tumor; thus, it provides improved tumor cure. The usage of IMRT has been very beneficial in terms of the optimization of radiotherapy (dose increase for complex shaped target volume, decrease of risky organ dose, providing the dose homogeneity, etc.) [1].

IMRT is an improved version of 3-D conformal therapy. Conformal dose distribution in IMRT is obtained with the help of multileaf collimator (MLC) in the head of linear accelerator. In contrast to the conventional or classical conformal therapy, dose intensity of each IMRT field is changed in a complex way. IMRT is applied in two ways: the first one is the dynamic IMRT (DMLC) technique formed by continuous movement of collimator leafs during irradiation, the second one is the step and shoot technique in which the beams are given by dividing into sub segments (SMLC) [1-3].

IMRT fields used to obtain conformal dose distribution comprise of small sub fields. The sizes of these fields might be $1 \times 1 \mathrm{~cm}^{2}$ sometimes. These small fields cause dosimetric problems such as sudden dose declines inside the therapy field. These rapid dose declines bring about divergence in dose that is required to be given to tumor.
Another dosimetric problem in small field usage is that the detectors used to take measurements are rather big volumetrically for the fields used in therapy. Standard detectors cannot exactly measure the dose value because of dose fluctuations in small field. Therefore, the ion chambers having smaller volume should be used $[4,5]$.

When Wolfram and Wong [6] have compared the measurements they have done with $0.6 \mathrm{cc}$ Farmer and 0.015 cc PinPoint ion chamber with the calculated values, they have seen that the ion chamber with $0.015 \mathrm{cc}$ has shown closer readings to the calculated values.

When they have compared these values to Thermoluminescence dosimetry (TLD) and diamond detector readings, the have found that the best result is the one read by diamond detector because it is the tissue equivalent and it has the perfect spatial resolution.

As seen from the conducted studies, different dosimetry systems can be used for IMRT quality reliability process. The important thing in the verification of IMRT is to use the most appropriate and accurate system.

The aim of this study is to investigate the most appropriate measurement method to overcome the dosimetric problems encountered in small field dosimetry which has an important place in intensity modulated radiotherapy.

\section{Material And Method}

Relative dose factors (RDF) of square fields between $1 \times 1-15 \times 15 \mathrm{~cm}$ formed by MLC in ARTISTE linear accelerator (6-15MV) device have been calculated by using differentvolume ion chambers and TLD. The results obtained by the used methods have been compared. Relative dose factors have been

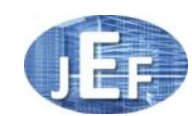


compared to the data of treatment planning system.

Whereas the primary dose constituting the dose in one point (primary dose+scattered dose) is independent from the field size, the scattered dose is dependent on the field size and beamed phantom size.

Therefore, relative dose factors have been found in order to show the dependence of the dose on field size. To find the factors, measurements have been made in $10 \mathrm{~cm}$ depth in $1 \times 1,2 \times 2,3 \times 3,4 \times 4,5 \times 5,6 \times 6,7 \times 7,8 \times 8,9 \times 9$, $10 \times 10$ ve $15 \times 15 \mathrm{~cm}$ fields in $15 \mathrm{MV}$ photon energy. The measurements have been made by giving $100 \mathrm{MU}$ in RW3 solid water phantom in center line and they have been repeated at least 3 times. The reading values have been normalized to the reading value of $10 \times 10 \mathrm{~cm}$ reference field and relative dose factors have been found and the graphic has been drawn to field width. Besides, dose efficiency measurement have been repeated in the same set-up conditions with TLD. Relative absorbed dose values have been examined according to the volumes of ion chambers. The results have been compared to the data of CMS XIO treatment planning system.

The measurements of solid water phantom have been made by using RW3 solid water phantom in center line for $15 \mathrm{MV}$ photon energy in $\mathrm{d}=$ $10 \mathrm{~cm} ; 1 \times 1,2 \times 2,3 \times 3,4 \times 4,5 \times 5,6 \times 6,7 \times 7$, $8 \times 8,9 \times 9,10 \times 10$ and $15 \times 15 \mathrm{~cm}$ field and using Farmer in SSD $100 \mathrm{~cm}\left(0,6 \mathrm{~cm}^{3}\right)$, Semiflex $\left(0,125 \mathrm{~cm}^{3}\right)$, PinPoint $\left(0,015 \mathrm{~cm}^{3}\right)$, Markus PP ion chambers $\left(0,055 \mathrm{~cm}^{3}\right)$ and PTW Unidos electrometer. $100 \mathrm{MU}$ has been given for each irradiation. The specially designed plate of solid water phantom has been used for each ion chamber in measurements. Each measurement has been repeated three times and the average has been calculated.
By placing TLD in center lines of , $2 \times 2,3 \times 3$, $4 \times 4,5 \times 5,6 \times 6,7 \times 7,8 \times 8,9 \times 9,10 \times 10$ and $15 \times 15$ cmfields in $15 \mathrm{MV}$ photon energy, measurement has been made by giving $100 \mathrm{MU}$ in $10 \mathrm{~cm}$ depth. The measurements have been repeated at least three times. The reading values have been normalized to the reading value of $10 \times 10 \mathrm{~cm}$ reference field.

TLDs that have been grouped and given numbers have been placed in TLD reader by keeping at least 10 hours after irradiation without ruining the sequencing. After the process of reading done by entering the numbers of each TLD one by one, radiant curves have been formed.

Reference point doses have been recorded by forming $2 \times 2,3 \times 3,4 \times 4,5 \times 5,6 \times 6,7 \times 7,8 \times 8$, $9 \times 9,10 \times 10$ and $15 \times 15 \mathrm{~cm}$ fields in $\mathrm{d}=1,5 \mathrm{~cm}$ and $\mathrm{d}=3 \mathrm{~cm}$ respectively in $15 \mathrm{MV}$ photon energy in CMS XIO.

\section{Findings}

\subsection{The results of measurements made by ion chamber}

Measurements have been taken by giving $100 \mathrm{MU}$ in $\mathrm{d}=10 \mathrm{~cm}$ in $1 \times 1,2 \times 2,3 \times 3$, $4 \times 4,5 \times 5,6 \times 6,7 \times 7,8 \times 8,9 \times 9,10 \times 10$ and $15 \times 15$ $\mathrm{cm}$ fields in $15 \mathrm{MV}$ photon energy by using Farmer, Semiflex , PinPoint and Markus PP ion chambers. The plans of these fields have been formed in CMS XIO and reference point doses have been recorded.

\subsection{The results of measurement made by $T L D$}

Measurements have been taken by giving $100 \mathrm{MU}$ in $\mathrm{d}=10 \mathrm{~cm}$ in $1 \times 1,2 \times 2,3 \times 3$, $4 \times 4,5 \times 5,6 \times 6,7 \times 7,8 \times 8,9 \times 9,10 \times 10$ and $15 \times 15$ $\mathrm{cm}$ fields in $15 \mathrm{MV}$ photon energy by using TLD. The plans of these fields have been 
formed in CMS XIO and reference point doses have been recorded.

\subsection{The results of measurement made by BTPS}

The results of reference point doses in $2 \times 2,3 \times 3,4 \times 4,5 \times 5,6 \times 6,7 \times 7,8 \times 8,9 \times 9,10 \times 10$ and $15 \times 15 \mathrm{~cm}$ formed in CMS XIO in $\mathrm{d}=1,5$ $\mathrm{cm}$ and $\mathrm{d}=3 \mathrm{~cm}$ respectively in $15 \mathrm{MV}$ photon energy have been given in the table below.

Table 1.BTPS data in $15 \mathrm{MV}$ photon energy in various square fields.

\begin{tabular}{|l|l|l|}
\cline { 2 - 3 } \multicolumn{1}{c|}{} & \multicolumn{1}{l|}{$\begin{array}{l}\text { ENERGY } \\
\text { 15 MV }\end{array}$} \\
\hline $\begin{array}{l}\text { FIELD } \\
\text { cm }^{2}\end{array}$ & READINGS & RDF \\
\hline 2X2 & 73,3 & 0,949 \\
\hline $3 \times 3$ & 74,5 & 0,965 \\
\hline $4 \times 4$ & 75,5 & 0,977 \\
\hline $5 \times 5$ & 75,8 & 0,981 \\
\hline $6 \times 6$ & 76,4 & 0,989 \\
\hline $7 \times 7$ & 76,9 & 0,996 \\
\hline $8 \times 8$ & 77,1 & 0,998 \\
\hline $9 \times 9$ & 77,2 & 1 \\
\hline $10 X 10$ & 77,2 & 1 \\
\hline $15 X 15$ & 77,9 & 1,009 \\
\hline
\end{tabular}

When 15 MV photon energy has been examined for Relative dose factors, the results of measurement found by using Farmer, Semiflex, PinPoint and Markus PP ion chambers with TLD have been demonstrated in Figure 1. The measurements made in $10 \mathrm{~cm}$ depth $(\mathrm{SSD}=100 \mathrm{~cm})$ have been normalized to 10x10 field dose. The plans of the same fields have been formed in CMS XIO in order to compare relative dose factors.

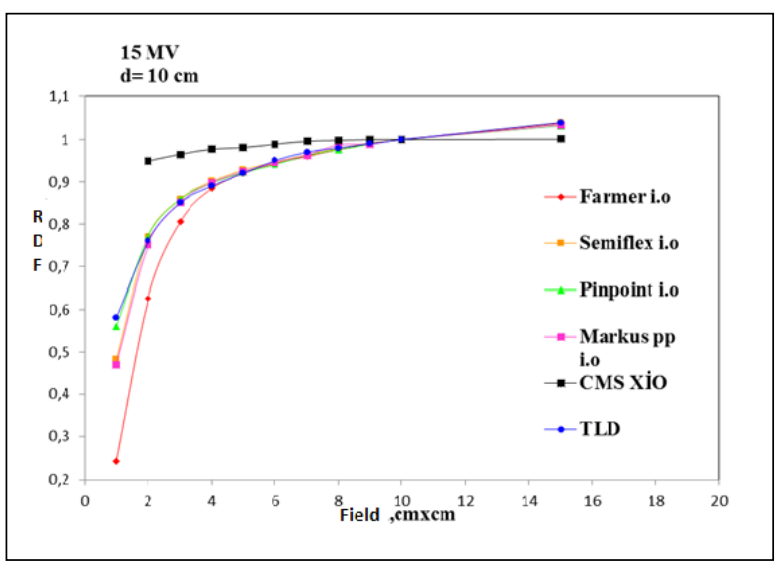

Figure1. Different ion chambers for $15 \mathrm{MV}$, The relative dose values with TLD and BTPS

It has been seen that Farmer ion chamber has done the lowest reference dose in $15 \mathrm{MV}$. The reason of it depends on the fact that Farmer ion chamber has bigger precise volume and it is also because of the breakdown in electron flow.

The relative dose factors that the computer treatment planning system has given in $15 \mathrm{MV}$ have been compared for the bigger fields than $2 \times 2 \mathrm{~cm}$ because the smallest field entered in the system is $2 \times 2 \mathrm{~cm}$. relative dose factors of computer treatment planning system are compatible with TLD results and ion chambers except for Farmer ion chamber.

\section{Results and Discussion}

The dose defined by IMRT is given by using multi small sub fields. In IMRT based on MLC, most of the fields are given from as small fields as $1 \times 1 \mathrm{~cm}$. Accordingly, small fields and their dosimetric properties should be modelled appropriately in TPS for an accurate dose calculation in IMRT. This study has been conducted to research different dosimetric methods in the verification of dose distribution of which relative dose factors have been modelled in TPS and to compare them and to find the most effective verification method in terms of accuracy and process easiness.

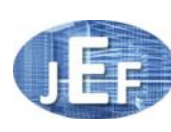


In the study, relative dose factors for $15 \mathrm{MV}$ photon energy in CMS XIO, TLD, Farmer, Semiflex, PinPoint and Markus PP ion chambers have been demonstrated in Figure 1.

Whereas PinPoint ion chamber and TLD readings have similar results in small fields for 15 MV photon energy, they give higher dose response than the other ion chambers. Semiflex ion chamber and Markus PP ion chamber are close to each other and they give lower dose response than PinPoint ion chamber and TLD readings. Farmer ion chamber shows severe dose decline than other ion chambers. This decline has some reasons. These are;

(1) The precise volume of Semiflex, Farmer and Markus PP ion chamber is bigger.

(2) Because of the breakdown in electron flow, the decline in electron flow ends in lower dose response by the decrease in ionization inside the ion chamber volume.

(3) The decline in the number of photon reaching the detector because of the spreading of electrons moving away from the center line and the decline of scattered dose. Maximum field dependence is seen in larger fields than the maximum distance of reaching of secondary electrons.

As seen in the study, the main reason why RDFs have been found smaller than $4 x 4 \mathrm{~cm}$ is that the lateral electron unbalance has increased by the increase of the measurement volume of the detector. It has been shown by similar studies that this increase in lateral electron unbalance might cause errors in dose calculations [7].

According to these evaluations, it has been inferred that the adequate use of small volume ion chamber in small field dosimetry will give

Journal of Engineering and Fundamentals (JEF) compatible results with film dosimetry having high spatial resolution and tissue equivalent TLD not forming perturbation factor. Besides, while low dose measurement caused by small volume ion chamber and lateral electron unbalance has been decreased, effective measurement point has been provided to take place inside the measurement fields. This situation will bring less uncertainty than bigger volume ion chamber. Similarly, Wolfram and Wong [6] have stated that the effect of the volume of ion chambers increase as the irradiated area grows in absolute dose measurements they have made with PintPoint $(0.015 \mathrm{cc})$ and $0.125 \mathrm{cc}$ ion chamber. They have noted that as the volume of the detector increases lateral electron unbalance increases and the read value decreases.

Martens et al. [8] have compared the measurements by using Markus PP ion chamber, PinPoint ion chamber and diamond detector. One of the findings of their study is that PinPoint chamber has given excessive reaction to low energy Compton scattering photons as in radiographic film. It depends on the fact that center electrode of PinPoint ion chamber is steel. They have recommended this ion chamber for out put measurements in 1.5 $\mathrm{cm}$ and smaller fields.

The measurements of relative dose factors of small field photon beams depend on the efficiency of the detectors used [9]. It has been revealed in many studies that diode and diamond detectors in relative dose factor have given the best results for small fields in many studies [10-12]. Besides, diamond detectors are very precise and expensive. Diode type should be chosen carefully because it might affect diode armor measurements negatively. Sanchez et al. [13] have compared the measurements by using a large field Markus PP, PinPoint, 
Semiflex, Farmer ion chambers, p type diode, diamond detector and Kodak X-Omat Vfilm. In the study, it has been seen that the volume precision of the detectors in output factors plays an important role. It has been reported that the precise volume of $0.6 \mathrm{~cm}^{3}$ ion chamber falls short of measuring the absorbed dose in small fields. Calcina et al. [4] have reached the conclusion that high-resolution film and TLDs are appropriate for small radiation fields in their studies in which they have evaluated the dosimetric parameters and various dosimetries. It has been noted that large volume ion chambers are not appropriate in high dose gradient fields because of the perturbation of electronic balance and volume average. Similarly, Laub and Wong [7] have notified that $0.015 \mathrm{~cm}^{3}$ volume PinPoint ion chamber has shown closer readings to the calculated values when they have compared the measurements they have made with $0.6 \mathrm{~cm}^{3}$ Farmer ion chamber and $0.015 \mathrm{~cm}^{3}$ PinPoint ion chamber with the calculated values. Westermark et al. [15] have informed that TLD relative dose factor measurements have shown closer readings to the calculated values than big volume ion chambers.

Pappas et al. [12] have measured the output factors of the field with $5 \mathrm{~mm}$ radius by using gel dosimetry. the average dose value between one voxel and zero voxel has been calculated inferentially by using the effect of volume and different voxel sizes. These results have coincided with the data of PinPoint ion chamber. It has been found out that ion chambers used routinely $\left(0,6 \mathrm{~cm}^{3}\right.$ volume $)$ in our study falls short of measuring the absorbed dose in small fields of precise volume. TLD and PinPoint ion chambers in small field RDF have shown similar readings. These results have been found compatible with the literature [14-17].

Considering the results of our study and similar studies in the literature, it is essential to choose the dosimetry volume as appropriate to field size and lateral electron unbalance we have encountered while evaluating the dosimetric parameters of small fields. It is necessary to choose the possible lowest volume and high spatial resolution dosimetry to decrease the effect of both factors. It should be paid attention that the dosimetrys have high spatial resolution in the small field photon therapy.

Whereas the average volume of dosimetry size and the presence of lateral electronic uncertainties and the uncertainties have minimized, the precise volume of the detector should be low enough in terms of saving the dose on shard drop area of the distribution. Besides, dosimetry should provide a spatial map of dose distribution and the accuracy of conformal distribution than the dose in a single point.

\section{References}

[1]. Wiezorek T, Banz N, Schwedas M, Scheithauer M, Salz H, Georg D, Wendt TG.Dosimetric quality assurance for intensity-modulated radiotherapy. Strahlentherapie und Oncology 2005;18: 468-474.

[2]. Letournea D, Gulam M, Yan D, Oldham M, Wong JW. Evaluation of a 2D diode array for IMRT quality assurance.Radiotherapy

Oncology2004;70: 199-206.

[3]. Wiezorek T, Schwedas M, Scheithauer M, Salz H, Bellemann M, Wendt TG.A new tool for quality assurance for intensity modulated radiotherapy.Strahlentherapie und Oncology2002; 178: 732-736.

[4]. Engler MJ, Hones G. Small beam calibration by $0,6-0,2$ cc ionization

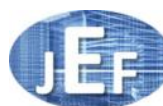


chamber.Medical Physics1984; 11:82226.

[5]. Wua Z, Kalend RD, Zheng AM. Comments on the dose measurements for a narrow beam radiosurgery.Medical Physic 1993; 20: 777-779.

[6]. Wolfram U, Wong T. The volume effect of detectors in the dissymmetry of small fields used in IMRT.Medical Physics2003; 30: 341-347.

[7]. Laub WU, Wong T.The volume effect of detectors in the dosimetry of small fields used in IMRT. Medical Physics2003; 30: 341-347.

[8]. Martens C, Wagter C, Deneve W. The value of the Pinpoint ion chamber for characterization of small field segments used in intensity-modulated radiotherapy. Physical Medical Biology2000; 45: 2519-2530.

[9]. Mckerracher C, Thwaites DI. Assessment of new small field detectors against standard field detectors for practical stereotactic beam data acquisition.Physical Medical Biology1999; 44: 2143-2160.

[10]. Devlamynck K, Palmans H, Verhaegen F, Thierens H.Dose measurements compared with Monte Carlo simulations of narrow $6 \mathrm{MV}$ multileaf collimator shaped photon beams.Medical. Physics1999; 26: 9-15.

[11]. Palta JR, Yeung DK, Frouhar V. Dosimetric considerations for a multileaf collimator system. Medical Physics 1996; 23: 1219-24.

[12]. PappasE, Marig TG, Papadakis A, Zacharopoulou F, Damilakis J. Experimental determination of the effect of detector size on profile measurements in narrow photon beams.Medical Physics1999; 26: 3700-3711.

[13]. Sanchez F, Hartmann GH, Pena J, Rosello JV, Russillo G, Gonzalez DM.A new method for output factor determination in MLC shapednarrow beam.Physical Medical2007; 23: 58-66.

[14]. Calcina CS, Oliveria LN, Almeida CE, Almeida A. Dosimetric parameters for small field sizes using fricke xylenol gel, thermoluminescent and film dosimeters and ionization chamber.Physical Medical Biology2007; 52: 1431-1439.

[15]. Westermark M, Amdt J, Nilsson B, Brahme A. Comparative dosimetry in narrow high-energy photon beams.Physical Medical Biology2000; 45: 685-702.

[16]. Wysocka A, Maciszewski W. The photon beam characteristics of lineer accelerator equipped with additional narrow beam collimator proceeding of EPAC.Physical Medical 2000; 10: 2563-2565.

[17]. DAS JI, DING XG, AHNESJO A. Small fields: no equilibrium radiation dosimetry.Medical Physics2008; 35: 206214. 